\title{
Targeted Nonlinear Energy Transfer for Electroacoustic Absorbers
}

\author{
D. Bitar, A. Ture Savadkoohi, C.-H. Lamarque, E. Gourdon and M. Collet
}

\begin{abstract}
In order to investigate the effects of coupling a nonlinear electrical shunt circuit to a loudspeaker terminal, a representative two degrees of freedom (dof) system has been considered. It consists of a main system describing the displacement of the loudspeaker membrane, which is linearly coupled to a Nonlinear Energy Sink (NES) with a small mass compared to the principal one. An analytical treatment enabling the analysis of the behaviour of the system around the 1:1 resonance at different time scales is endowed. This methodology enables the detection of equilibrium and singular points, corresponding to periodic and modulated regimes, respectively. The analytical developments prepare necessary design tools for tuning parameters of the NES.
\end{abstract}

Keywords Electroacoustic absorber · Passive control $\cdot$ Nonlinear energy sink Targeted energy transfer

D. Bitar $(\bowtie) \cdot$ A. Ture Savadkoohi $\cdot$ C.-H. Lamarque $\cdot$ E. Gourdon LTDS-UMR CNRS 5513, Ecole Nationale des Travaux Publics de l'Etat (ENTPE), Univ. Lyon, 3 rue Maurice Audin, 69120 Vaulx-en-Velin Cedex, France e-mail: diala.bitar@entpe.fr
A. Ture Savadkoohi
e-mail: alireza.turesavadkoohi@entpe.fr
C.-H. Lamarque
e-mail: Claude-Henri.Lamarque@entpe.fr
E. Gourdon
e-mail: emmanuel.gourdon@entpe.fr

M. Collet

LTDS-UMR CNRS 5513, Ecole Centrale de Lyon (ECL), 36 avenue Guy de Collongue, 69134 Ecully Cedex, France

e-mail:manuel.collet@ec-lyon.fr 


\subsection{Introduction}

Audible sound is a combination of direct sound flowing from a source and indirect reflections. In order to improve the quality of sound in a room, the control of noise reverberations at the propagation and reception paths is essential. Among the various employed sound absorption technologies, we are interested in the active absorption approach. For instance, an electrodynamic loudspeaker can be turned into an electroacoustic absorber by connecting a convenient passive electrical shunt circuit to the transducer terminal. This approach permits to dissipate sound power of incident waves [1]. The concept of energy pumping from a primary source to a NES was introduced in several domains of engineering sciences with two main applications namely passive control and energy harvesting (see for example [2-4]).

Nonlinear systems are well known for their ability to improve the performance of the control and increasing frequency range of absorption [5]. For this purpose, a passive nonlinear shunt circuit has been connected to the loudspeaker terminal. Then, the whole structure can be represented by a two dof system, including the main linear system describing the displacement of the loudspeaker membrane which is linearly coupled to an electrical NES.

In order to solve the system analytically, an extended version of complex variables of Manevitch [6] is introduced, taking into account higher harmonics. It permits a better prediction of system behaviors, especially during bifurcations. The multiplescale method [7] is employed enabling to detect the behavior of the system at different time scales. This approach allows the identification of the system invariant at fast time scale and equilibrium and singular points at slow times scales [8, 9].

\subsection{System Representation}

The dynamics of an electroacoustic loudspeaker, shunted with an electrical nonlinear circuit and subjected to an external periodically varying sound pressure can be described by the following differential system:

$$
\left\{\begin{array}{l}
M_{m s} \ddot{x}(t)+R_{m s} \dot{x}(t)+C_{m c}^{-1} x(t)-C B l \dot{V}(t)=S A_{m} \cos (\Omega t) \\
C\left(L_{e}+L_{c}\right) \ddot{V}(t)+C\left(R_{e}+R_{c}\right) \dot{V}(t)+k V^{3}+B l \dot{x}(t)=0
\end{array}\right.
$$

where $x$ and $V$ describe respectively the small displacement of the loudspeaker membrane and the electric potential in the nonlinear shunt circuit. The dot notation indicates the derivative with respect to time $t$ i.e. $\dot{x}=d x / d t . M_{m s}, R_{m s}$ and $C_{m c}$ are the mass, the mechanical resistance of the moving bodies and the equivalent compliance of the enclosed loudspeaker. $B l$ is the force factor of the transducer with $B$ representing the magnetic field magnitude and $l$ standing for the length of the wire in the voice coil. $A_{m}$ is the pressure amplitude, $\Omega$ is the angular frequency and $S$ stands for the diaphragm surface. $R_{e}$ and $L_{e}$ are respectively the DC resistance 
and the inductance of the voice coil with $B l \dot{x}(t)$ describing the back electromotive force. $R_{c}, L_{c}$ and $C$ are the inductance, resistance, and capacitance of the corresponding nonlinear shunt circuit with $k$ the nonlinear coefficient related to the multiplier connections.

After introducing the non-dimensional time variable $T=\omega_{0} t$ with $\omega_{0}=$ $\sqrt{1 /\left(M_{m s} C_{m c}\right)}$ the natural angular frequency, the physical two degree of freedom system of Eqs. (7.1) can be expressed by the following scaled system

$$
\left\{\begin{array}{l}
\ddot{y}_{1}+\varepsilon \lambda \dot{y}_{1}+y_{1}-\varepsilon \alpha \dot{y}_{2}=\varepsilon f \cos (\omega T), \\
\varepsilon\left(\ddot{y}_{2}+\gamma \dot{y}_{2}+\xi y_{2}^{3}+\eta \dot{y}_{1}\right)=0 .
\end{array}\right.
$$

The dot notation indicates now the derivative with respect to time $T . y_{1}$ and $y_{2}$ stand for $x$ and $V$ in the new time domain. The scaled parameters used in Sys. (7.2) are $\varepsilon=L_{e}+L_{c}, R_{m s} C_{m c} \omega_{0}=\varepsilon \lambda, C B l C_{m c} \omega_{0}=\varepsilon \alpha, S A_{m} C_{m c}=\varepsilon f,\left(R_{e}+R_{c}\right) / \omega_{0}=$ $\varepsilon \gamma, k /\left(C \omega_{0}^{2}\right)=\varepsilon \xi, \omega=\Omega / \omega_{0}$ and $B l /\left(C \omega_{0}\right)=\varepsilon \eta$.

\subsection{Analytical Treatment}

In order to analyze the dynamical behavior of the system around the $1: 1$ resonance by letting $\omega=1+\varepsilon \sigma$ with $\sigma$ a detuning parameter, Manevitch's complex variables [6] can be introduced as

$$
\left\{\begin{array}{l}
\dot{y}_{1}+i \omega y_{1}=\psi_{1}(T) e^{i \omega T} \\
\dot{y}_{2}+i \omega y_{2}=\psi_{2}(T) e^{i \omega T}
\end{array}\right.
$$

Before introducing the complex variables (7.3) into the scaled Sys. (7.2), we choose to investigate on the contribution of harmonics in both variables $\dot{y}_{n}+i \omega y_{n}$ with $n \in\{1,2\}$. Then, we plot in Fig. 7.1 the modulus of $\dot{y}_{1}+i \omega y_{1}$ and $\dot{y}_{2}+i \omega y_{2}$ according to scaled time $T$ for the following parameters: $\varepsilon=0.01, \eta=\alpha=\lambda=0.2$, $\gamma=0.3, \xi=0.5$ and $f=0$. The corresponding numerical results obtained by direct numerical integration of Eqs. (7.2) using a Runge-Kutta scheme with the corresponding initial conditions as $y_{1}(0)=y_{2}(0)=\dot{y}_{2}(0)=0$ and $\dot{y}_{1}(0)=3.7$.

Remarkably, for the first modulus represented in Fig. 7.1a, it can be clearly seen that the first harmonic is sufficient enough to qualify the energy level of the primary system. Thus, for the energy amplitude of the NES represented in Fig. 7.1b the addition of the third harmonic has remarkable effects on its behavior the individual presence of the first harmonic.

\subsubsection{Dynamical Behavior Around 1:1 Resonance}

An extended version of Manevitch's complex variables is introduced in the present study, taking into account the effect of the first harmonic for the principal system and 

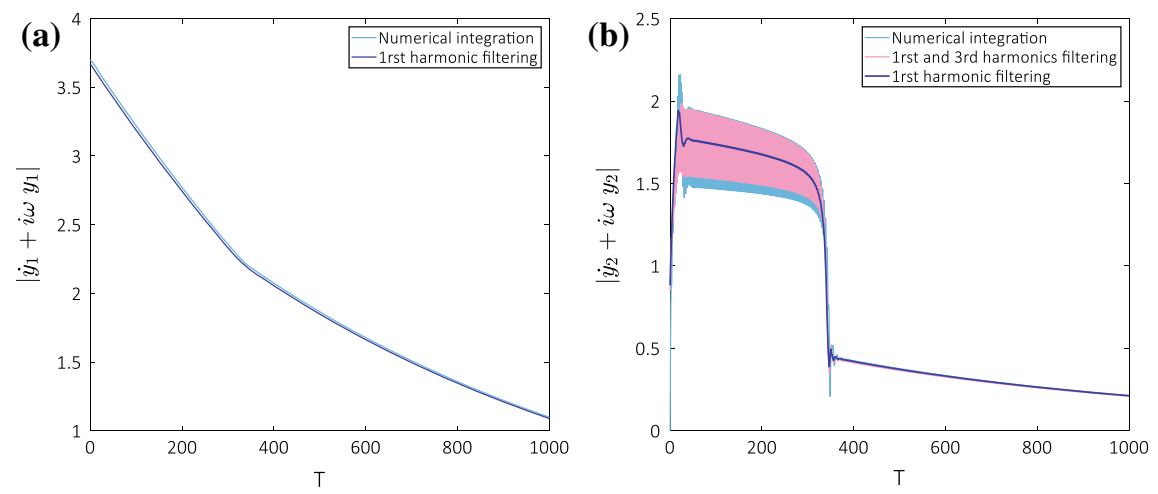

Fig. 7.1 Time histories of $\left|d y_{1} / d T+i \omega y_{1}\right|=N_{1}$ and $\left|d y_{2} / d T+i \omega y_{2}\right|$ obtained by the direct integration of Sys. (7.2) under free oscillations; i.e. $f=0$

both first and third harmonics for the NES. In addition, we apply a multiple scales approach by introducing a fast time scale $\tau_{0}$ and slower ones $\left(\tau_{1}, \tau_{2}, \ldots\right)$ as:

$$
T=\tau_{0}, \tau_{1}=\varepsilon \tau_{0}, \tau_{2}=\varepsilon^{2} \tau_{0} \ldots
$$

The new complex variables are introduced as in the following relationship:

$$
\left\{\begin{array}{l}
\dot{y}_{1}+i \omega y_{1}=\psi_{1}\left(\tau_{1}, \tau_{2}, \ldots\right) e^{i \omega \tau_{0}} \\
\dot{y}_{2}+i \omega y_{2}=\psi_{2}\left(\tau_{1}, \tau_{2}, \ldots\right) e^{i \omega \tau_{0}}+\psi_{3}\left(\tau_{1}, \tau_{2}, \ldots\right) e^{3 i \omega \tau_{0}}
\end{array}\right.
$$

After introducing the complex variables presented in Eq. (7.5) into Eqs. (7.2) we obtain the system below

$$
\left\{\begin{array}{c}
{\left[\dot{\psi}_{1}-i \frac{\left(1-\omega^{2}\right)}{2 \omega} \psi_{1}+\varepsilon \frac{\lambda}{2} \psi_{1}-\varepsilon \frac{\alpha}{2} \psi_{2}-\varepsilon \frac{f}{2}\right] e^{i \omega \tau_{0}}-\varepsilon \frac{\alpha}{2} \psi_{3} e^{3 i \omega \tau_{0}}+c . c .=0} \\
{\left[\dot{\psi}_{2}+i \frac{\omega}{2} \psi_{2}+\frac{\gamma}{2} \psi_{2}+\frac{\eta}{2} \psi_{1}-i \frac{3 \xi}{8 \omega^{3}}\left(\psi_{2}\left|\psi_{2}\right|^{2}-\psi_{3} \psi_{2}^{*^{2}}+2 \psi_{2}\left|\psi_{3}\right|^{2}\right)\right] e^{i \omega \tau_{0}}} \\
+\left[\dot{\psi}_{3}+i \frac{5 \omega}{2} \psi_{3}+\frac{\gamma}{2} \psi_{3}-i \frac{\xi}{8 \omega^{3}}\left(3 \psi_{3}\left|\psi_{3}\right|^{2}-\psi_{2}^{3}+6 \psi_{3}\left|\psi_{2}\right|^{2}\right)\right] e^{3 i \omega \tau_{0}} \\
+i \frac{3 \xi}{8 \omega^{3}} \psi_{2} \psi_{3}^{2} e^{7 i \omega \tau_{0}}+i \frac{3 \xi}{8 \omega^{3}}\left[\psi_{2}^{2} \psi_{3}^{3}-\psi_{3}^{2} \psi_{2}^{*}\right] e^{5 i \omega \tau_{0}}+\text { c.c. }=0
\end{array}\right.
$$

where c.c. stands for the complex conjugate of the rest of the arguments. The complex variables $\psi_{n}$ describe the slow modulation of fast vibrations at the frequency $\omega$.

Using the Galerkin technique [9], we keep the first harmonic of the main system and the first and the third harmonics of the NES and truncate higher ones. In applying the method, we suppose that $\psi_{1}, \psi_{2}, \psi_{3}$ are independent of the fast time $\tau_{0}$. Then, we obtain an averaged system composed of three first order differential equations in terms of $\psi_{1}, \psi_{2}$ and $\psi_{3}$ as: 


$$
\begin{aligned}
& \dot{\psi}_{1}-i \frac{\left(1-\omega^{2}\right)}{2 \omega} \psi_{1}+\varepsilon \frac{\lambda}{2} \psi_{1}-\varepsilon \frac{\alpha}{2} \psi_{2}=\varepsilon \frac{1}{2} f \\
& \dot{\psi}_{2}+i \frac{\omega}{2} \psi_{2}+\frac{\gamma}{2} \psi_{2}+\frac{\eta}{2} \psi_{1}-i \frac{3 \xi}{8 \omega^{3}}\left(\psi_{2}\left|\psi_{2}\right|^{2}-\psi_{3} \psi_{2}^{*^{2}}+2 \psi_{2}\left|\psi_{3}\right|^{2}\right)=0 \\
& \dot{\psi}_{3}+i \frac{5 \omega}{2} \psi_{3}+\frac{\gamma}{2} \psi_{3}-i \frac{\xi}{8 \omega^{3}}\left(3 \psi_{3}\left|\psi_{3}\right|^{2}-\psi_{2}^{3}+6 \psi_{3}\left|\psi_{2}\right|^{2}\right)=0
\end{aligned}
$$

This methodology enables the detection of the system invariant at the fast time scale $\tau_{0}$, which allows the detection of the system behaviors at the slower time scales.

\subsubsection{The System Behavior at $\tau_{0}$ Time Scale}

At the order $\varepsilon^{0}$, resonant terms at $\tau_{0}$ time scale in Eq. (7.7) give

$$
\frac{\partial \psi_{1}}{\partial \tau_{0}}=0 \Rightarrow \psi_{1}=\psi_{1}\left(\tau_{1}, \tau_{2}, \ldots\right)
$$

Then $\psi_{1}$ is constant according to the fast time scale $\tau_{0}$ winch validates our hypotheses during using the Galerkin method. However, Eqs. (7.8) and (7.9) can be expressed as in the following form

$$
\left\{\begin{array}{l}
\frac{\partial \psi_{2}}{\partial \tau_{0}}+H_{1}\left(\psi_{1}, \psi_{2}, \psi_{3}, \psi_{2}^{*}, \psi_{3}^{*}\right)=0 \\
\frac{\partial \psi_{3}}{\partial \tau_{0}}+H_{2}\left(\psi_{2}, \psi_{3}, \psi_{2}^{*}, \psi_{3}^{*}\right)=0
\end{array}\right.
$$

where $H_{1}$ and $H_{2}$ define the $\varepsilon^{1}$ order functions of Eqs. (7.8) and (7.9). System (7.11) presents an asymptotic equilibrium governed by a manifold called Slow Invariant Manifold (SIM), which is in fact a geometrical representation of the fixed points of the system i.e.,

$$
\tau_{0} \longrightarrow \infty \Rightarrow\left\{\begin{array}{l}
\frac{\partial \psi_{2}}{\partial \tau_{0}} \longrightarrow 0 \\
\frac{\partial \psi_{3}}{\partial \tau_{0}} \longrightarrow 0
\end{array}\right.
$$

or we can set

$$
\mathscr{H}\left(\delta_{1}, N_{1}, \delta_{2}, N_{2}, \delta_{3}, N_{3}\right)=0 \equiv\left\{\begin{array}{l}
H_{1}\left(\psi_{1}, \psi_{2}, \psi_{3}, \psi_{2}^{*}, \psi_{3}^{*}\right)=0 \\
H_{2}\left(\psi_{2}, \psi_{3}, \psi_{2}^{*}, \psi_{3}^{*}\right)=0
\end{array}\right.
$$

Writing the complex variables in the polar form as $\psi_{j}=N_{j} e^{i \delta_{j}}$ with $j=1,2,3$, Sys. (7.13) can be expressed and reduced to the following form after separating its real and imaginary parts: 


$$
\begin{aligned}
h_{1}= & N_{2}\left[\gamma+\frac{3}{4} \xi \sin \left(3 \delta_{2}-\delta_{3}\right) N_{2} N_{3}\right]+\eta \cos \left(\delta_{1}-\delta_{2}\right) N_{1}=0 \\
h_{2}= & N_{2}\left[1-\frac{3}{4} \xi\left(N_{2}\left(N_{2}+\cos \left(3 \delta_{2}-\delta_{3}\right) N_{3}\right)-2 N_{3}^{2}\right)\right] \\
& \quad+\eta \sin \left(\delta_{1}-\delta_{2}\right) N_{1}=0 \\
h_{3}= & 4 \gamma N_{3}-\xi \sin \left(3 \delta_{2}-\delta_{3}\right) N_{2}^{3}=0 \\
h_{4}= & 4 N_{3}\left[-5+\frac{3}{2} \xi N_{2}^{2}+\frac{3}{4} \xi N_{3}^{2}\right]-\xi \cos \left(3 \delta_{2}-\delta_{3}\right) N_{2}^{3}=0
\end{aligned}
$$

Replacing Eqs. (7.16) and (7.17) into Eqs. (7.14) and (7.15) respectively, the SIM can be expressed as it follows:

$$
\left\{\begin{array}{l}
N_{1}=\frac{N_{2}}{\eta} \sqrt{\gamma^{2}\left(1+3 \frac{N_{3}^{2}}{N_{2}^{2}}\right)^{2}+\left(1-\frac{3}{4} \xi N_{2}^{2}-15 \frac{N_{3}^{2}}{N_{2}^{2}}+3 \xi N_{3}^{2}+\frac{9}{4} \xi \frac{N_{3}^{4}}{N_{2}^{2}}\right)^{2}} \\
\xi^{2} N_{2}^{6}=16 N_{3}^{2}\left[\gamma^{2}+\left(-5+\frac{3}{2} \xi N_{2}^{2}+\frac{3}{4} \xi N_{3}^{2}\right)^{2}\right] .
\end{array}\right.
$$

Under free oscillations, we plot in Fig. 7.2 the numerical result obtained by a direct integration of the scaled Sys. (7.2) compared to the SIM obtained by solving Sys. (7.18). The classical SIM driven by employing the first harmonics only is represented in dashed dot line can be obtained after replacing $N_{3}$ by 0 in Eq. (7.18). Remarkably, the addition of the third harmonic managed to adjust the gap between the numerical integration and analytical developments, mainly at the bifurcation.

Fig. 7.2 A comparison between the classical $(---)$ and modified $(*)$ SIM together with the direct numerical integration (__ ) of the scaled Sys. (7.2) under free oscillations; i.e. $f=0$

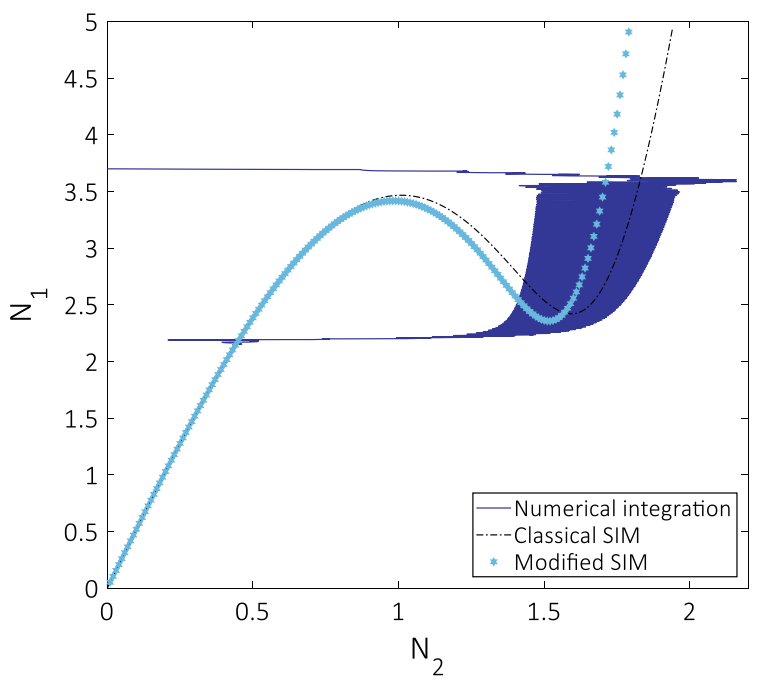




\subsubsection{The System Behavior at $\tau_{1}$ Time Scale}

Treating Eq. (7.7) at the order $\varepsilon^{1}$, we can analytically identify the equilibrium points and singularities leading to the presence of periodic or quasi-periodic regimes. Equation (7.7) at $\varepsilon^{1}$ reads:

$$
\frac{\partial \psi_{1}}{\partial \tau_{1}}=f\left(\psi_{1}, \psi_{2}\right)=-\left(\frac{\lambda}{2}+i \sigma\right) \psi_{1}+\frac{\alpha}{2} \psi_{2}+\frac{f}{2}
$$

Writing Eq. (7.19) into its polar form and separating its real and imaginary parts we obtain the following system

$$
\left\{\begin{array}{l}
\frac{\partial \delta_{1}}{\partial \tau_{1}}=f_{1}\left(\delta_{1}, N_{1}, \delta_{2}, N_{2}\right)=-\frac{\lambda}{2} N_{1}+\frac{\alpha}{2} N_{2} \cos \left(\delta_{2}-\delta_{1}\right)+\frac{f}{2} \cos \left(\delta_{1}\right) \\
\frac{\partial N_{1}}{\partial \tau_{1}}=f_{2}\left(\delta_{1}, N_{1}, \delta_{2}, N_{2}\right)=\frac{1}{N_{1}}\left(\frac{\alpha}{2} N_{2} \sin \left(\delta_{2}-\delta_{1}\right)-\sigma N_{1}-\frac{f}{2} \sin \left(\delta_{1}\right)\right) .
\end{array}\right.
$$

Combining Eqs. (7.13) and (7.20), the system behavior at slow time scale $\tau_{0}$ around its invariant can be studied using following equation

$$
\left(\begin{array}{llllll}
\frac{\partial h_{1}}{\partial \delta_{1}} & \frac{\partial h_{1}}{\partial N_{1}} & \frac{\partial h_{1}}{\partial \delta_{2}} & \frac{\partial h_{1}}{\partial N_{2}} & \frac{\partial h_{1}}{\partial \delta_{3}} & \frac{\partial h_{1}}{\partial N_{3}} \\
\frac{\partial h_{2}}{\partial \delta_{1}} & \frac{\partial h_{2}}{\partial N_{1}} & \frac{\partial h_{2}}{\partial \delta_{2}} & \frac{\partial h_{2}}{\partial N_{2}} & \frac{\partial h_{2}}{\partial \delta_{3}} & \frac{\partial h_{1}}{\partial N_{3}} \\
\frac{\partial h_{3}}{\partial \delta_{1}} & \frac{\partial h_{3}}{\partial N_{1}} & \frac{\partial h_{3}}{\partial \delta_{2}} & \frac{\partial h_{3}}{\partial N_{2}} & \frac{\partial h_{3}}{\partial \delta_{3}} & \frac{\partial h_{3}}{\partial N_{3}} \\
\frac{\partial h_{4}}{\partial \delta_{1}} & \frac{\partial h_{4}}{\partial N_{1}} & \frac{\partial h_{4}}{\partial \delta_{2}} & \frac{\partial h_{4}}{\partial N_{2}} & \frac{\partial h_{4}}{\partial \delta_{3}} & \frac{\partial h_{4}}{\partial N_{3}}
\end{array}\right)\left(\begin{array}{l}
\frac{\partial \delta_{1}}{\partial \tau_{1}} \\
\frac{\partial N_{1}}{\partial \tau_{1}} \\
\frac{\partial \delta_{2}}{\partial \tau_{1}} \\
\frac{\partial N_{2}}{\partial \tau_{1}} \\
\frac{\partial \delta_{3}}{\partial \tau_{1}} \\
\frac{\partial N_{3}}{\partial \tau_{1}}
\end{array}\right)=\left(\begin{array}{l}
0 \\
0 \\
0 \\
0 \\
0 \\
0
\end{array}\right)
$$

Equation(7.21) can be arranged to be written in the following form

$$
\underbrace{\left(\begin{array}{llll}
\frac{\partial h_{1}}{\partial \delta_{2}} & \frac{\partial h_{1}}{\partial N_{2}} & \frac{\partial h_{1}}{\partial \delta_{3}} & \frac{\partial h_{1}}{\partial N_{3}} \\
\frac{\partial h_{2}}{\partial \delta_{2}} & \frac{\partial h_{2}}{\partial N_{2}} & \frac{\partial h_{2}}{\partial \delta_{3}} & \frac{\partial h_{1}}{\partial N_{3}} \\
\frac{\partial h_{3}}{\partial \delta_{2}} & \frac{\partial h_{3}}{\partial N_{2}} & \frac{\partial h_{3}}{\partial \delta_{3}} & \frac{\partial h_{3}}{\partial N_{3}} \\
\frac{\partial h_{4}}{\partial \delta_{2}} & \frac{\partial h_{4}}{\partial N_{2}} & \frac{\partial h_{4}}{\partial \delta_{3}} & \frac{\partial h_{4}}{\partial N_{3}}
\end{array}\right)}_{\mathscr{M}}\left(\begin{array}{l}
\frac{\partial \delta_{2}}{\partial \tau_{1}} \\
\frac{\partial N_{2}}{\partial \tau_{1}} \\
\frac{\partial \delta_{3}}{\partial \tau_{1}} \\
\frac{\partial N_{3}}{\partial \tau_{1}}
\end{array}\right)=-\left(\begin{array}{ll}
\frac{\partial h_{1}}{\partial \delta_{1}} & \frac{\partial h_{1}}{\partial N_{1}} \\
\frac{\partial h_{2}}{\partial h_{2}} & \frac{\partial h_{2}}{\partial N_{1}} \\
0 & 0 \\
0 & 0
\end{array}\right)\left(\begin{array}{l}
f_{1}\left(\delta_{1}, N_{1}, \delta_{2}, N_{2}\right) \\
f_{2}\left(\delta_{1}, N_{1}, \delta_{2}, N_{2}\right)
\end{array}\right)
$$


Then, equilibrium points of the system, can be obtained by solving the following system [10]

$$
\left\{\begin{array}{l}
\mathscr{H}=0 \\
f_{1}=f_{2}=0 \\
\operatorname{det}(\mathscr{M}) \neq 0
\end{array}\right.
$$

However, fold singularities are reached when

$$
\left\{\begin{array}{l}
\mathscr{H}=0 \\
f_{1}=f_{2}=0 \\
\operatorname{det}(\mathscr{M})=0
\end{array}\right.
$$

\subsection{Some Results and Discussion}

Let us consider a system with $f=0.3$ and the detuning parameter $\sigma=0$, Sys. (7.23) is validated with $f_{1}=f_{2}=0, \mathscr{H}=0$ and $\operatorname{det}(\mathscr{M}) \neq 0$, which indicates the existence of an equilibrium point. Figure 7.3a shows the modified SIM compared to the numerical integration of the scaled Sys. (7.2). This later oscillates around the upper branch of the SIM, then once reaching the stability border, it jumps to follow the small amplitude level to be finally attracted by an equilibrium point. The existence of an equilibrium point indicates the existence of a periodic regime, which is verified in Fig. 7.3b once reaching the permanent regime.

Solving Sys. (7.23) for $f=0.3$ and $\sigma=0$, we can deduce the existence of the equilibrium point $\left(N_{2}, N_{1}\right)=(0.274,1.3959)$. Then, we can deduce that the analytical predictions are in good agreement with the numerical results depicted in Fig. 7.4,
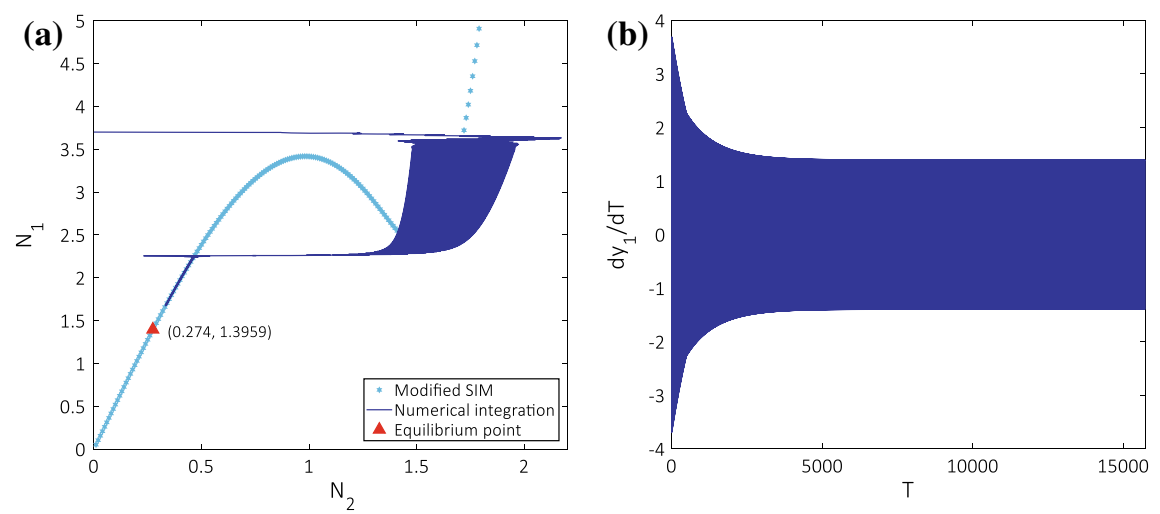

Fig. 7.3 a SIM of the system with the numerical integration of the scaled Sys.(7.2) under small forced oscillations with $f=0.3$ and the detuning parameter $\sigma=0$. b Time histories of the velocity $d y_{1} / d T$ 

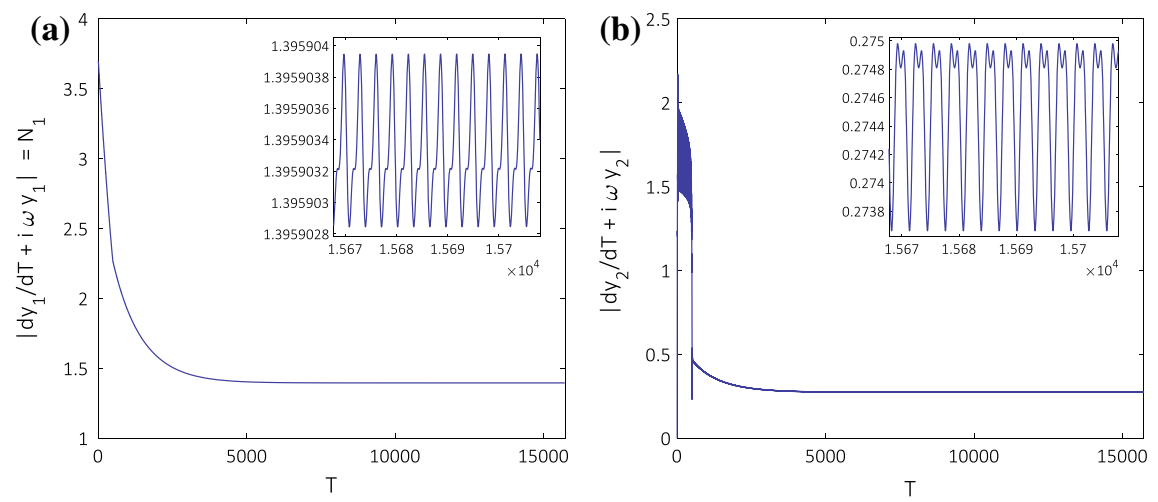

Fig. 7.4 Time histories of both amplitudes $\left|d y_{1} / d T+i \omega y_{1}\right|=N_{1}$ and $\left|d y_{2} / d T+i \omega y_{2}\right|$ obtained by the direct integration of Sys. (7.2) under small forced oscillations with $f=0.3$ and the detuning parameter $\sigma=0$

where we plot the histories of $N_{1}=\left|\frac{d y_{1}}{d T}+i \omega y_{1}\right|$ and $\left|\frac{d y_{2}}{d T}+i \omega y_{2}\right|$. In this given example, the NES is able to control the primary system against periodic external forces by presenting small amplitudes during periodic regimes.

\subsection{Conclusions}

In order to reduce noise at the propagation and reception paths, an electroacoustic loudspeaker has been turned into a passive absorber by coupling to its terminal a nonlinear electrical shunt circuit. The nonlinear behavior of the system was described by a rescaled two degrees of freedom system, which consists of a linear master structure under sinusoidal forcing that is linearly coupled to a nonlinear energy sink. The study was carried out using an extended version Manevitch's complex variables, including first and third harmonics. The complex system was treated analytically using a multiple-scale method, allowing the detection of the system invariant at the fast time scale. A predictive tool enabling the identification of the dynamical regime (periodic or modulated regimes) is given for the purpose of passive control of the main system.

Acknowledgements This work was conducted in the framework of the LABEX CeLyA ("Centre Lyonnais d'Acoustique"), ANR-10-LABX-60. 


\section{References}

1. Lissek, H., Boulandet, R., Fleury, R.: Electroacoustic absorbers: bridging the gap between shunt loudspeakers and active sound absorption. JASA 129, 2968-2978 (2011)

2. Gourdon, E., Alexander, N.A., Taylor, C.A., Lamarque, C.-H., Pernot, S.: Nonlinear energy pumping under transient forcing with strongly nonlinear coupling: theoretical and experimental results. JSV 300, 522-551 (2007)

3. Bellet, R., Cochelin, B., Herzog, P., Mattei, P.O.: Experimental study of targeted energy transfer from an acoustic system to a nonlinear membrane absorber. JSV 329, 2768-2791 (2010)

4. Vakakis, A.F., Gendelman, O.V., Bergman, L.A., McFarland, D.M., Kerschen, G., Lee, Y.S.: Nonlinear Targeted Energy Transfer in Mechanical and Structural Systems. Springer, Netherlands (2009)

5. Roberson, R.E.: Synthesis of a nonlinear dynamic vibration absorber. J. Franklin Inst. 254, 205-220 (1952)

6. Manevitch, L.I.: The description of localized normal modes in a chain of nonlinear coupled oscillators using complex variables. Nonlinear Dyn. 25, 95-109 (2001)

7. Nayfeh, A., Mook, D.: Nonlinear Oscillations. Wiley, New York (1979)

8. Weiss, M., Chenia, M., Ture Savadkoohi, A., Lamarque, C.-H., Vaurigaud, B., Hammouda, A.: Multi-scale energy exchanges between an elasto-plastic oscillator and a light nonsmooth system with external pre-stress. Nonlinear Dyn. 83, 109-135 (2016)

9. Lamarque, C.-H., Ture Savadkoohi, A.: Dynamical behavior of a Bouc Wen type oscillator coupled to a nonlinear energy sink. Meccanica 49, 1917-1928 (2014)

10. Ture Savadkoohi, A., Lamarque, C.-H., Weiss, M., Vaurigaud, B., Charlemagne, S.: Analysis of the 1:1 resonant energy exchanges between coupled oscillators with rheologies. Nonlinear Dyn. 86, 2145-2159 (2016) 